\title{
Review of the Clinical Applications of Trabecular Bone Score
}

\author{
Hossein Yarmohammadi ${ }^{1,2}$, Alireza Mirzaei ${ }^{3}$, Mozhdeh Zabihiyeganeh ${ }^{3^{*}}$ \\ ${ }^{1}$ Medical Students Research Committee, Shahed University, Tehran, Iran. ${ }^{2}$ Osteoporosis Research Center, Endocrinology \\ and Metabolism Clinical Sciences Institute, Tehran University of Medical Sciences, Tehran, Iran. ${ }^{3}$ Bone and Joint \\ Reconstruction Research Center, Shafa Orthopedic Hospital, Iran University of Medical Sciences, Tehran, Iran.
}

\begin{abstract}
Assessment of bone health is essential in many different diseases and conditions, including osteoporosis and fragility fracture. Although bone mineral density (BMD) has a well-established role in measuring bone quantity, it is unable to determine bone quality. Recently, the trabecular bone score (TBS) was developed as a valuable approach to determining bone quality by evaluating the microarchitecture of a bone. TBS data is of critical importance, particularly in conditions in which BMD is falsely elevated or has not yet decreased significantly. The current review has collected current evidence regarding the clinical applications of TBS in various disorders, such as osteoporosis, fracture, diabetes, chronic kidney disease, and degenerative bone diseases.
\end{abstract}

Keywords: Bone, Microarchitecture, Osteoporosis, Trabecular bone score

\section{Introduction}

Bone mineral density (BMD) is a valuable tool for determining bone quantity, but it is flawed by the inability to assess bone quality. Trabecular bone score (TBS) is a new bone evaluating measure that provides valuable information about bone microarchitecture[1]. It is an analytical tool that quantifies variation in grey-level texture on the standard lumbar spine BMD[2]. This feature allows better evaluation of bone status, thereby easing therapeutic decision-making for responsible physicians. Combining TBS with the fracture risk assessment tool (FRAX) has also been successful in estimating the risk of fracture [3]. Due to the importance of bone quality in the prediction of fracture risk, TBS is continuously drawing the attention of professionals in the assessment of bone health. In particular, it is suggested for use in conditions in which BMD is not capable of accurately measuring bone health, such as in patients with degenerative bone diseases [4].

The excitement of researchers and clinicians has led to an increasing number of publications about the application of TBS in a variety of diseases and conditions. This article aims to review the wide range of TBS applications in medical conditions.

\section{TBS Definition}

TBS is captured from the gray-level variation of pixels on DXA images as a higher number, and the homogeneity of these pixels indicates a better microarchitecture and bone quality [5] (Figure 1). Although the TBS range for normal and abnormal values is still an open research question, there is greater consensus on the following [1]:

Normal (not degraded): $>1.31$

Borderline (partially degraded): $\leq 1.31$ and $>1.23$

Abnormal (degraded): $\leq 1.23$

Nevertheless, a recent study on an Iranian population has suggested microarchitecture degradation cut-offs of $\leq 1.23$ and $\leq 1.29$ in men and women, respectively [6].

\section{Fracture Prediction}

FRAX is an online system developed by the World Health Organization (WHO) that is used extensively to estimate the probability of future fragility fractures. Despite the valuable information it provides, until recently, it was only based on the mineral density of bone. Therefore, a great deal of information regarding the bone quality was missed. After the development of TBS, FRAX incorporated the TBS data into BMD information, thereby providing a fracture risk assessment based on both bone quality and quantity. This feature allows a more accurate estimation of bone health and provides better identification of at-risk populations requiring therapeutic intervention for fracture prevention [7]. The literature review suggests that

Personal non-commercial use only. Rheumatology Research Journal. Copyright $($ C 2020. All rights reserved

*Corresponding Author: Mozhdeh Zabihiyeganeh, Shafa Orthopedic Hospital, Baharestan Square, Tehran, Iran. PO-Box: +98(21)33542000-8, E-mail: zabihiyeganeh.m@iums.ac.ir, mozhdehzabihi@gmail.com, Telefax: +98(21)33542020.

Received: 16 November 2019; Accepted: 30 January 2020 
TBS alone is capable of fracture prediction independent of BMD. However, its combination with BMD results in a more accurate assessment. Moreover, recent evidence suggests that TBS may even be superior to FRAX in fracture prediction in situations where BMD alone is unreliable and not of great help [8].

A recent study in Japan on more than 1500 adults and a follow-up of 10 years concluded that TBS score alone could be used for fracture prediction, and adding TBS to FRAX resulted in a more accurate fracture prediction [9]. The meta-analysis of McCloskey et al. on a pooled population of 17,809 adults concluded that TBS could predict fracture independently of FRAX, and when it is used conjunct with FRAX, the accuracy of prediction is increased [10].

The study of Mirzaei et al. on 358 postmenopausal Iranian women did not show any significant clinical benefit of TBS adjusted FRAX on improving fracture prediction or therapeutic decision-making. They concluded that TBS evaluation is more valuable for patients with borderline risk of major osteoporotic and hip fracture, as the choice of therapeutic intervention in these patients is more probable to change after FRAX adjustment with TBS. In addition, their study showed that TBS evaluation is of great value for patients with severe osteophytes in their spine X-rays, because BMD results are falsely normal in these patients [11].

Holloway et al. reported similar findings on the value of FRAX adjustment with TBS in an Australian population [12]. Future research will provide more precise evidence for the TBS application in daily routine practice and will identify the population who will take the most benefit from this tool. Moreover, TBS measurement shows technical variations, and with the newly published study of Shafiee et al. on the reference value of TBS in an Iranian population, further research is needed [6].

\section{Body Mass Index (BMI) Effect on TBS}

TBS is directly affected by abdominal soft tissue that influences the grey-level pixels, and thus, TBS is negatively affected by fat tissue. Langsetmo et al. revealed that the association between BMD and TBS is affected by BMI. They recommended limiting the application of TBS in men with BMI higher than $37 \mathrm{~kg} / \mathrm{m}^{2}$, particularly those with abdominal obesity [13]. Conversely, Shayganfar et al. studied 1054 postmenopausal women in Iran and concluded that BMI had no significant association with TBS[14]. Interestingly, Kim et al. reported that although higher BMI was related to lower TBS both in men and women, height was directly associated with TBS in women, and weight was directly associated with TBS in men [15]. This might be due to the different patterns of fat tissue accumulation in men and women. Central obesity is more prevalent in men than in women, and abdominal fat tissue may significantly influence TBS obtained from lumbar spine BMD.

\section{TBS Application in Diabetes}

Diabetes mellitus (DM) adversely affects bone health with several mechanisms such as vasculopathy, inflammation, hyperglycemia, and altered metabolism. Reduced bone turnover and microarchitecture degradation are eventually presented as bone fragility [16]. Although epidemiologic studies suggest that diabetics, especially elderly people, are more prone to osteoporosis and fractures [17], other studies have reported that BMD in DM patients is not reduced and is even above the normal average [18-20]. Still other studies suggest that diabetes mostly affects bone quality and not its density [21]. In that case, TBS evaluation in diabetic patients would be of critical value, and for this reason, interest in applying TBS in DM cases is increasing.

In several studies, TBS has been successful in assessing bone quality and in predicting fracture risk in DM patients $[22,23]$. It has also been shown to be negatively correlated with DM risk factors, including high hemoglobin A1c and fasting glucose [24]. In a recent meta-analysis of 12 studies with a pooled population of 4962 men and 35,546 women, TBS was significantly lower in diabetics, and the difference was greater in women [25]. In addition, TBS was significantly lower in prediabetics compared to the healthy population [25], which may be related to microarchitecture degradation as a key mechanism in DM [21]. Despite the result of this meta-analysis and other original studies, some investigations have reported that TBS was not significantly different between diabetics and healthy people $[24,26]$. This discrepancy may be attributable to the difference in DM duration and the therapeutic regimen used by patients [25]. Overall, BMD and FRAX are tools of great help in osteoporosis and fracture management for DM patients, and TBS could improve these tools [27]. Future studies will provide a more precise understanding of the role of TBS in DM patients.

TBS Application in Chronic Kidney Disease (CKD) and Kidney Transplant Recipients (KTR)

Osteoporosis and fractures are prevalent in CKD patients, and guidelines recommend BMD as a routine clinical practice for assessing bone health in these patients [28]. However, BMD is not reliable enough for fracture prediction in these patients compared to healthy people [29], mostly due to calcifications of joints and arthritic vasculatures [30]. For this reason, imaging methods that provide information on bone microarchitecture, including magnetic resonance imaging (MRI), high-resolution peripheral quantitative computed tomography (HRpQCT), and TBS, are now under investigation to enhance current bone densitometry techniques. TBS has been shown to be significantly reduced in end-stage renal disease (ESRD) patients [31-34] and has successfully predicted fractures in CKD patients [33, 35]. Furthermore, it has been reported to be correlated with estimated glomerular filtration rate (eGFR); lower TBS was seen in patients with eGFR $\leq 60 \mathrm{~mL} / \mathrm{min} / 1.73 \quad \mathrm{~m}^{2} \quad$ [36]. Nevertheless, the application of TBS in CKD patients resulted in a significant alteration in the number of patients receiving osteoporosis treatment [37]

Aortic calcification in KTR patients is an important cause of falsely elevated BMD. Luckman et al. concluded 
that TBS was superior to spine BMD in predicting fractures and could successfully monitor the loss of bone quality in the first year following transplantation. These observations reveal that TBS may be of considerable value to reporting early bone loss in KTR patients, when the reduced quantity of bone is not yet detectable on BMD [38]. However, there is an evidence gap on this research topic, and further largescale prospective studies are needed to investigate the change of TBS following a kidney transplant in early and long-term follow-up. Still, the current evidence suggests that TBS may be of use in KTR patients [39].

TBS Application in Ankylosing Spondylitis (AS) and Rheumatoid Arthritis (RA)

Arthritis adversely affects bone, cartilage, and joints, and the presence of osteophytes and falsely increases BMD syndesmophytes. However, since this osteoproliferation affects only the quantity and not the quality of bone, TBS provides a more accurate measurement of bone health in these patients [40]. According to Bréban et al., TBS successfully detected vertebral fractures among RA patients, and the number of these fractures was associated with lower TBS [41]. Based on the report of Choi et al., this association is even more prominent in RA patients receiving glucocorticoids [42]. Kim et al. reported that TBS more precisely predicts fractures compared to BMD, especially in RA patients receiving glucocorticoids [43] Nam et al. reported that TBS is useful in predicting fractures and measuring bone quality, even with the presence of osteoproliferation [44]. Other studies have also report that TBS accurately diagnosed axial osteoporosis in the early stages of AS, while BMD is not a reliable tool. Moreover, TBS is reported to be capable of predicting spine fractures independent of FRAX in AS patients [4547].

\section{TBS Application in Osteoarthritis (OA)}

TBS application in OA patients is not yet well studied. Kolta et al. revealed that patients with lumbar spine OA have a higher BMD than those without it. In contrast, spine TBS is not affected by the presence of OA. This data suggested a more reliable implication of TBS than BMD in patients with lumbar spine OA [48]. The study of Padlina et al. on a cohort of 1443 women revealed that despite the increase in BMD at the age of 62.5 years, TBS drops each year. Accordingly, they concluded that TBS is not influenced by degenerative diseases of bone [49]. Although the evidence of TBS application in OA is still limited, interest in TBS in future research will increase, because TBS is not influenced by spondylosis [50].

\section{TBS and Treatment with Glucocorticoids}

Glucocorticoids are among the most prescribed medications worldwide and a major cause of secondary osteoporosis with a significant risk of fracture in the longterm [51]. BMD is valuable in providing a baseline measurement before the initiation of glucocorticoid therapy, and FRAX could be used to estimate the initial risk of fracture. However, despite a daily dose of glucocorticoids, the length of the treatment and the cumulative dose is not considered in this algorithm [52].
Since microarchitecture degradation is one of the mechanisms involved in glucocorticoid-induced osteoporosis [53], TBS may be a beneficial tool for the evaluation of bone health in this population. Nonetheless, evidence of TBS application for glucocorticoid-induced osteoporosis management is limited, and there are discrepancies among the published articles. One study reports that TBS predicts and diagnoses fractures, especially vertebral fractures, in glucocorticoid-induced osteoporosis [54]. Other studies, however, report TBS as not being capable of fracture risk assessment independently, but having added value when used in combination with FRAX [55]. TBS is capable of monitoring treatment response; because glucocorticoids mostly reduce bone formation, anabolic agents (teriparatide) show more desirable clinical outcomes in glucocorticoid-induced osteoporosis compared to antiresorptive agents (alendronate). Thus, TBS could be a sensitive tool in distinguishing between different treatments [56]. Overall, TBS adjunct to FRAX is useful for fracture prediction in patients receiving glucocorticoids. However, future studies are needed to shed more light on TBS application in the management of glucocorticoid-induced osteoporosis.

\section{TBS Application in Bariatric Surgery}

Bariatric surgery is an effective treatment for morbid obesity, yet it has many potential long-term complications. This surgery adversely affects bone health through the nutritional and hormonal changes that result in reduced bone turnover and degraded microarchitecture [57]. Current evidence suggests that BMD alone is not a precise measure for bone changes after bariatric surgery, and quantitative computed tomography (QCT) or HR-pQCT are studied to predict fracture risk. However, there is limited evidence regarding the application of TBS for such purpose [58]. In one randomized clinical trial with two years of follow-up, TBS was reduced during the first six months after bariatric surgery and then remained unchanged after interventions such as vitamin D and exercise. However, the change in BMD between intervention and observation groups was not significant [59]. Another study revealed similar findings after three years of follow-up, although TBS did not change FRAX data significantly [60].

\section{TBS Application in Breast Cancer}

Aromatase inhibitors (AIs) are among first-line therapies in breast cancer, but they may cause osteoporosis and increase the risk of fracture by reducing bone turnover [61]. TBS, as an adjunct to BMD, has been investigated by some researchers for screening osteoporosis and its management in breast cancer patients under treatment with AIs. According to these studies, TBS improves BMD and FRAX evaluation in these patients; therefore, adding TBS to FRAX could be helpful in maximizing the identification of at-risk patients before, during, or after AIs treatment [6264].

Application of TBS in patients undergoing spine surgery 
Patients with degenerative lumbar spine pathology who are eligible for spinal fusion surgery are at high risk of postoperative fracture. The use of BMD alone to diagnose osteoporosis in patients with lumbar spondylosis is not reliable and has provoked interest in additional methods of evaluating preoperative spinal bone health. Jeffery et al. aimed to determine which patients should be considered for preoperative bone health optimization to reduce the risk of postoperative fracture. According to this study, of the patients not identified with osteoporosis using BMD, $70.4 \%$ had osteoporosis with expanded spine criteria, which included TBS. The authors suggested an expanded spine definition considering the TBS data to more comprehensively identify patients with poor bone health that could benefit from preoperative optimization. Nevertheless, this implication of TBS, similar to its other implications, needs further validation in future researches [50].

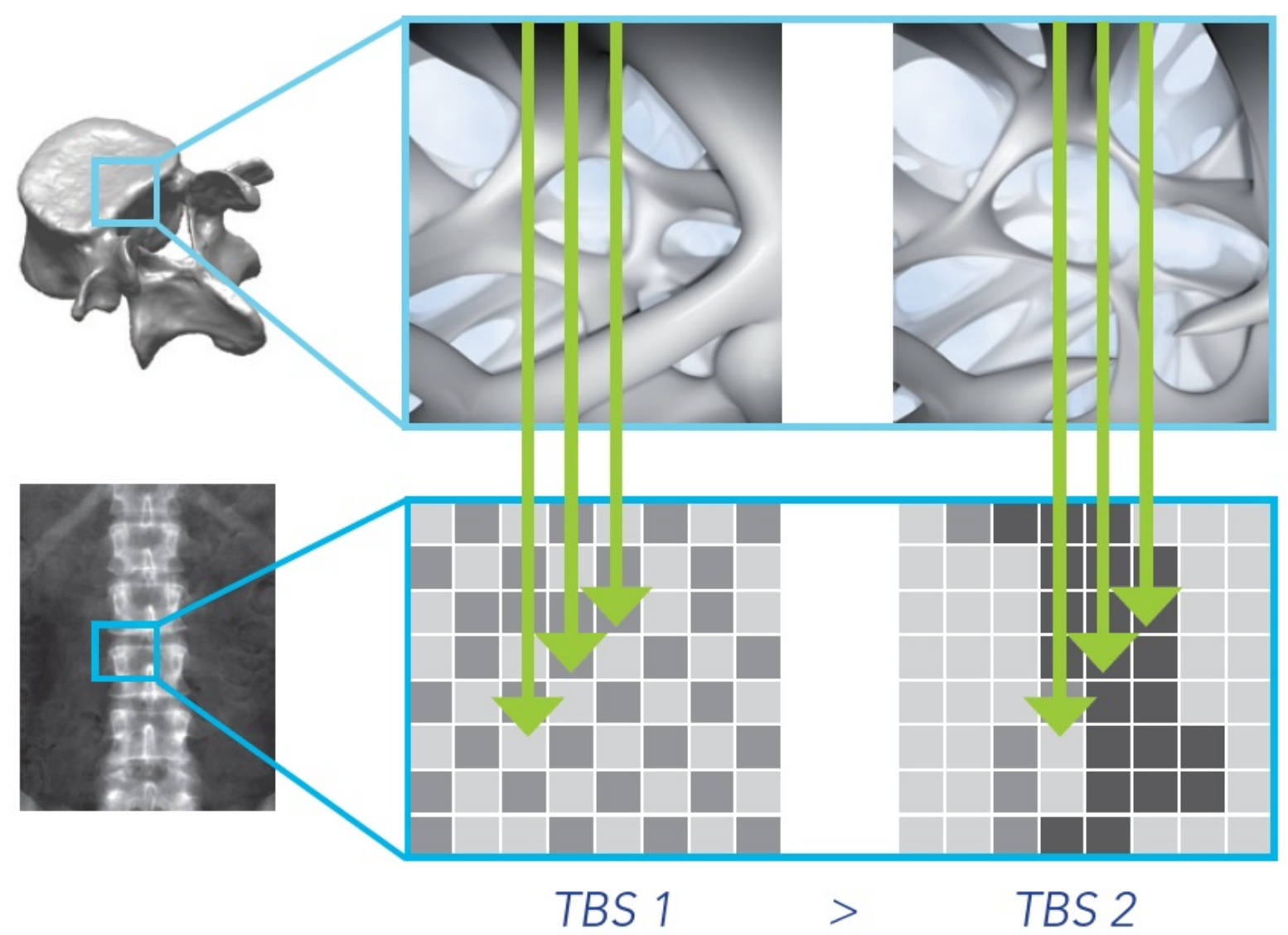

Figure 1: Comparison of normal (TBS1) and altered (TBS2) trabecular structure of bone (https://www.roentgen-baden.at)

\section{Conclusion}

Generally, TBS is useful for a variety of conditions, at least adjunct to BMD and FRAX. However, in some conditions such as osteoarthritis, TBS would be of more value, because the results of BMD are misleading. Future complementary research is needed to shed more light on the gaps in knowledge and to translate the application of TBS from research into clinical implications. While TBS is expected to attract more interest for the management of secondary osteoporosis, such as in ankylosing spondylitis, renal transplant recipients, or treatment with glucocorticoids, the limitations of TBS application should be properly addressed. For example, TBS is highly dependent on the quality of imaging, and conditions such as abdominal obesity may lead to false TBS results.

\section{Conflict of interest}

None to declare. 


\section{References}

1. Markovic-Temelkova S, Foteva M, Jovanovska ES. CLINICAL USE OF TRABECULAR BONE SCORE FOR THE DIAGNOSIS OF OSTEOPOROSIS: A REVIEW. J Morphol Sci 2019; 2(2):95-103.

2. Silva BC, Leslie WD, Resch H, Lamy O, Lesnyak O, Binkley N. et al. Trabecular bone score: a noninvasive analytical method based upon the DXA image. J Bone Miner Res 2014; 29(3):518-30. doi: 10.1002/jbmr.2176.

3. Martineau P, Leslie W. Trabecular bone score (TBS): Method and applications. Bone 2017; 104:66-72. doi: 10.1016/j.bone.2017.01.035.

4. Anderson KB, Holloway-Kew KL, Mohebbi M, Kotowicz MA, Hans D, Pasco JA. Is trabecular bone score less affected by degenerative-changes at the spine than lumbar spine BMD? Arch Osteoporos 2018; 13(1):127. doi: 10.1007/s11657-018-0544-3.

5. Silva BC, Leslie WD. Trabecular bone score: a new DXA-derived measurement for fracture risk assessment. Endocrinol Metab Clin North Am 2017; 46(1):153-80. doi: 10.1016/j.ecl.2016.09.005.

6. Shafiee G, Sharifi F, Heshmat R, Ostovar A, Ebrahimpur M, Sheidaei A. et al. The reference value of trabecular bone score (TBS) in the Iranian population. J Diabetes Metab Disord 2020; 19(1):493-98. doi: 10.1007/s40200-020-00537-w.

7. Couraud G, Souffir C, Gaigneux E, Kolta S, Roux C, Briot K. Adjusting FRAX ${ }^{\circledR}$ on TBS for identification of subjects at high risk of fractures. Bone 2017; 101:214-18. doi: 10.1016/j.bone.2017.05.005.

8. Hans D, Šteňová E, Lamy O. The trabecular bone score (TBS) complements DXA and the FRAX as a fracture risk assessment tool in routine clinical practice. Curr Osteoporos Rep 2017; 15(6):521-31. doi: 10.1007/s11914-017-0410-z.

9. Tamaki J, Iki M, Sato Y, Winzenrieth R, Kajita E, Kagamimori S. et al. Does Trabecular Bone Score (TBS) improve the predictive ability of FRAX ${ }^{\circledR}$ for major osteoporotic fractures according to the Japanese Population-Based Osteoporosis (JPOS) cohort study? J Bone Miner Metab 2019; 37(1):161-70. doi: 10.1007/s00774-018-0910-7.

10. McCloskey EV, Odén A, Harvey NC, Leslie WD, Hans D, Johansson H. et al. A Meta-Analysis of Trabecular Bone Score in Fracture Risk Prediction and Its Relationship to FRAX. J Bone Miner Res 2016; 31(5):940-48. doi: 10.1002/jbmr.2734.
11. Mirzaei A, Jahed SA, Nojomi M, Rajaei A, Zabihiyeganeh M. A study of the value of trabecular bone score in fracture risk assessment of postmenopausal women. Taiwan J Obstet Gynecol 2018; 57(3):389-93.doi: 10.1016/j.tjog.2018.04.011.

12. Holloway KL, Mohebbi M, Betson AG, Hans D, Hyde $\mathrm{N}$, Brennan-Olsen S. et al. Prediction of major osteoporotic and hip fractures in Australian men using FRAX scores adjusted with trabecular bone score. Osteoporos Int 2018; 29(1):101-08. doi: 10.1007/s00198-017-4226-6.

13. Langsetmo L, Vo TN, Ensrud KE, Taylor BC, Cawthon PM, Schwartz AV. et al. The association between trabecular bone score and lumbar spine volumetric BMD is attenuated among older men with high body mass index. J Bone Miner Res 2016; 31(10):1820-26. doi: 10.1002/jbmr.2867

14. Shayganfar A, Farrokhi M, Shayganfar S, Ebrahimian S. Associations between bone mineral density, trabecular bone score, and body mass index in postmenopausal females. Osteoporos Sarcopenia 2020; 6(3):111-14. doi: 10.1016/j.afos.2020.08.002.

15. Kim Y-S, Han J-J, Lee J, Choi HS, Kim JH, Lee T. The correlation between bone mineral density/trabecular bone score and body mass index, height, and weight. Osteoporos Sarcopenia 2017; 3(2):98-03. doi: 10.1016/j.afos.2017.02.001 .

16. Ghodsi M, Keshtkar AA, Nasli-Esfahani E, Alatab S, Mohajeri-Tehrani MR. Mechanisms involved in altered bone metabolism in diabetes: a narrative review. J Diabetes Metab Disord 2016; 15(1):52.doi: 10.1186/s40200-016-0275-1.

17. Poiana C, Capatina C. Fracture risk assessment in patients with diabetes mellitus. J Clin Densitom 2017; 20(3):432-43. doi: 10.1016/j.jocd.2017.06.011..

18. Kao WL, Kammerer CM, Schneider JL, Bauer RL, Mitchell BD. Type 2 diabetes is associated with increased bone mineral density in Mexican-American women. Arch Med Res 2003; 34(5):399-06. doi: 10.1016/j.arcmed.2002.07.001.

19. Gerdhem P, Isaksson A, Åkesson K, Obrant KJ. Increased bone density and decreased bone turnover, but no evident alteration of fracture susceptibility in elderly women with diabetes mellitus. Osteoporos Int 2005; 16(12):1506-12. doi: 10.1007/s00198-0051877-5. 
20. Dennison E, Syddall H, Sayer AA, Craighead S, Phillips D, Cooper C. Type 2 diabetes mellitus is associated with increased axial bone density in men and women from the Hertfordshire Cohort Study: evidence for an indirect effect of insulin resistance? Diabetologia 2004; 47(11):1963-68. doi: 10.1007/s00125-004-1560-y.

21. Jiang N, Xia W. Assessment of bone quality in patients with diabetes mellitus. Osteoporos Int 2018; 29(8):1721-36. doi: 10.1007/s00198-018-4532-7.

22. McCloskey EV, Odén A, Harvey NC, Leslie WD, Hans D, Johansson H. et al. A meta-analysis of trabecular bone score in fracture risk prediction and its relationship to FRAX. J Bone Miner Res 2016; 31(5):940-48. doi: 10.1002/jbmr.2734.

23. Martineau P, Leslie WD, Johansson H, Oden A, McCloskey EV, Hans D. et al. Clinical utility of using lumbar spine trabecular bone score to adjust fracture probability: the Manitoba BMD cohort. J Bone Miner Res 2017; 32(7):1568-74. doi: 10.1002/jbmr.3124.

24. Kim JH, Choi HJ, Ku EJ, Kim KM, Kim SW, Cho NH. et al. Trabecular bone score as an indicator for skeletal deterioration in diabetes. J Clin Endocrinol Metab 2015; 100(2):475-82. doi: 10.1210/jc.2014-2047.

25. Ho-Pham L, Nguyen T. Association between trabecular bone score and type 2 diabetes: a quantitative update of evidence. Osteoporos Int 2019; 30(10):2079-85. doi: 10.1007/s00198-019-05053-z.

26. Iki M, Fujita Y, Kouda K, Yura A, Tachiki T, Tamaki J. et al. Hyperglycemia is associated with increased bone mineral density and decreased trabecular bone score in elderly Japanese men: The Fujiwara-kyo osteoporosis risk in men (FORMEN) study. Bone 2017; 105:18-25. doi: 10.1016/j.bone.2017.08.007.

27. Leslie WD, Johansson H, McCloskey EV, Harvey NC, Kanis JA, Hans D. Comparison of methods for improving fracture risk assessment in diabetes: the Manitoba BMD Registry. J Bone Miner Res 2018; 33(11):1923-30. doi: 10.1002/jbmr.3538.

28. Ketteler M, Block GA, Evenepoel P, Fukagawa M, Herzog CA, McCann L. et al. Executive summary of the 2017 KDIGO chronic kidney disease-mineral and bone disorder (CKD-MBD) guideline update: what's changed and why it matters. Kidney Int 2017; 92(1):26-36. doi: 10.1016/j.kint.2017.04.006.

29. Negri AL, Barone R, Quiroga MA, Bravo M, Marino A, Fradinger E. et al. Bone mineral density: serum markers of bone turnover and their relationships in peritoneal dialysis. Perit dial int 2004; 24(2):163-68. doi.org/10.1177/089686080402400207.
30. Pimentel A, Ureña-Torres P, Zillikens MC, Bover J, Cohen-Solal M. Fractures in patients with CKDdiagnosis, treatment, and prevention: a review by members of the European Calcified Tissue Society and the European Renal Association of Nephrology Dialysis and Transplantation. Kidney Int 2017; 92(6):1343-55. doi: 10.1016/j.kint.2017.07.021.

31. Brunerova L, Ronová $\mathrm{P}$, Verešová $\mathrm{J}$, Beranova $\mathrm{P}$, Potoèková J, Kasalický P. et al. Osteoporosis and impaired trabecular bone score in hemodialysis patients. Kidney Blood Press Res 2016; 41(3):345-54. doi: 10.1159/000443439.

32. Yavropoulou MP, Vaios V, Pikilidou M, Chryssogonidis I, Sachinidou M, Tournis S. et al. Bone quality assessment as measured by trabecular bone score in patients with end-stage renal disease on dialysis. J Clin Densitom 2017; 20(4):490-97. doi: 10.1016/j.jocd.2016.11.002.

33. Aleksova J, Kurniawan S, Elder G. The trabecular bone score is associated with bone mineral density, markers of bone turnover and prevalent fracture in patients with end stage kidney disease. Osteoporos Int 2018; 29(6):1447-55. doi: 10.1007/s00198-018-4468-y.

34. Dusceac R, Niculescu DA, Dobre R, Dragne MC, Tacu C, Peride I. et al. Chronic hemodialysis is associated with lower trabecular bone score, independent of bone mineral density: a case-control study. Arch Osteoporos 2018; 13(1):125. doi: 10.1007/s11657-018-0541-6.

35. Naylor KL, Prior J, Garg AX, Berger C, Langsetmo L, Adachi JD. et al. Trabecular bone score and incident fragility fracture risk in adults with reduced kidney function. Clin J Am Soc Nephrol 2016; 11(11):203240. doi: 10.2215/CJN.00720116.

36. Rampersad C, Whitlock R, Leslie W, Rigatto C, Komenda P, Bohm C. et al. Trabecular bone score in patients with chronic kidney disease. Osteoporos Int 2020; 31(10):1905-12 doi: 10.1007/s00198-02005458-1.

37. Malakoutian T, Mirzaei A, Shiroudbakhshi A, Kadijani AA, Tehrani-Banihashemi A, Zabihiyeganeh M. The added value of trabecular bone score in fracture risk assessment of kidney transplant recipients. Iran J Kidney Dis 2020; 14(4):300-07.

38. Luckman M, Hans D, Cortez N, Nishiyama KK, Agarawal S, Zhang C. et al. Spine trabecular bone score as an indicator of bone microarchitecture at the peripheral skeleton in kidney transplant recipients. Clin J Ame Soc Nephrol 2017; 12(4):644-52. doi: 10.2215/CJN.09850916. 
39. Shevroja E, Lamy O, Hans D. Review on the utility of trabecular bone score, a surrogate of bone microarchitecture, in the chronic kidney disease spectrum and in kidney transplant recipients. Front Endocrinol 2018; 9:561. doi: 10.3389/fendo.2018.00561.

40. Magrey MN, Lewis S, Khan MA. Utility of DXA scanning and risk factors for osteoporosis in ankylosing spondylitis - a prospective study. Semin Arthritis Rheum 2016; 46(1) 88-94. doi: 10.1016/j.semarthrit.2016.03.003.

41. Bréban S, Briot K, Kolta S, Paternotte S, Ghazi M, Fechtenbaum J. et al. Identification of Rheumatoid Arthritis Patients with Vertebral Fractures Using Bone Mineral Density and Trabecular Bone Score. J Clin Densitom 2012; 15(3):260-66. doi: 10.1016/j.jocd.2012.01.007

42. Choi YJ, Chung Y-S, Suh C-H, Jung J-Y, Kim H-A. Trabecular bone score as a supplementary tool for the discrimination of osteoporotic fractures in postmenopausal women with rheumatoid arthritis. Medicine 2017; 96(45):e8661. doi: 10.1097/MD.0000000000008661.

43. Kim D, Cho S-K, Kim JY, Choi YY, Sung Y-K. Association between trabecular bone score and risk factors for fractures in Korean female patients with rheumatoid arthritis. Mod Rheumatol 2016; 26(4):540-45. doi: 10.3109/14397595.2015.1101212.

44. Nam SW, Sung Y-K, Kim D, Cho S-K, Song Y, Choi YY. et al. The usefulness of trabecular bone score in patients with ankylosing spondylitis. Korean J Int Med 2020. doi: 10.3904/kjim.2020.065.

45. Adel Y, El Adalany MA, Bassiouni SARAK. Performance of bone mineral density and trabecular bone score in assessment of bone quality in Egyptian male patients with ankylosing spondylitis. Egyptian Rheumatol Rehab 2020; 47(1):1-8. doi.org/10.1186/s43166-020-00036-Z.

46. Richards C, Hans D, Leslie WD. Trabecular Bone Score (TBS) Predicts Fracture in Ankylosing Spondylitis: The Manitoba BMD Registry. J Clin Densitom 2020; 23(4):543-48. doi: 10.1016/j.jocd.2020.01.003

47. Zabihiyeganeh M, Mirzaei A. The Value of Trabecular Bone Score in the Evaluation of Bone Quality in a Patient with Ankylosing Spondylitis. Shafa Orthoped J 2017; 4(3). doi.org/10.5812/soj.11450.

48. Kolta S, Briot K, Fechtenbaum J, Paternotte S, Armbrecht G, Felsenberg D. et al. TBS result is not affected by lumbar spine osteoarthritis. Osteoporos Int 2014; 25(6):1759-64. doi: 10.1007/s00198-0142685-6.
49. Padlina I, Gonzalez-Rodriguez E, Hans D, Metzger M, Stoll D, Aubry-Rozier B. et al. The lumbar spine agerelated degenerative disease influences the BMD not the TBS: the Osteolaus cohort. Osteoporos Int 2017; 28(3):909-15. doi: 10.1007/s00198-016-3829-7.

50. Jeor JDS, Jackson TJ, Xiong AE, Kadri A, Freedman BA, Sebastian AS. et al. Osteoporosis in spine surgery patients: what is the best way to diagnose osteoporosis in this population? Neurosurg Focus 2020; 49(2):E4 doi: $10.3171 / 2020.5$.FOCUS20277.

51. Adami G, Saag K. Glucocorticoid-induced osteoporosis: 2019 concise clinical review. Osteoporos Int 2019; 30(6):1145-56. doi: 10.1007/s00198-019-04906-x.

52. Kanis JA, Johansson H, Oden A, McCloskey EV. Guidance for the adjustment of FRAX according to the dose of glucocorticoids. Osteoporos Int 2011; 22(3):809-16. doi: 10.1007/s00198-010-1524-7.

53. Compston J. Glucocorticoid-induced osteoporosis: an update. Endocrine 2018; 61(1):7-16. doi: 10.1007/s12020-018-1588-2.

54. Florez H, Hernández-Rodríguez J, Muxi A, Carrasco JL, Prieto-González S, Cid MC. et al. Trabecular bone score improves fracture risk assessment in glucocorticoid-induced osteoporosis. Rheumatology (Oxford) 2020; 59(7):1574-80. doi: 10.1093/rheumatology/kez464.

55. Chuang M-H, Chuang T-L, Koo M, Wang Y-F. Trabecular Bone Score reflects trabecular microarchitecture deterioration and fragility fracture in female adult patients receiving glucocorticoid therapy: a pre-post controlled study. BioMed res Int 2017; 2017:4210217. doi: 10.1155/2017/4210217.

56. Saag KG, Agnusdei D, Hans D, Kohlmeier LA, Krohn KD, Leib ES. et al. Trabecular Bone Score in Patients With Chronic Glucocorticoid Therapy-Induced Osteoporosis Treated With Alendronate or Teriparatide. Arthritis Rheum 2016; 68(9):2122-28. doi: 10.1002/art.39726.

57. Beavers KM, Greene KA, Elaine WY. MANAGEMENT OF ENDOCRINE DISEASE: Bone complications of bariatric surgery: updates on sleeve gastrectomy, fractures, and interventions. Eur $\boldsymbol{J}$ Endocrinol 2020; 183(5):R119-R32. doi: 10.1530/EJE-20-0548.

58. Gagnon C, Schafer AL. Bone health after bariatric surgery. JBMR plus 2018; 2(3):121-33. doi: 10.1002/jbm4.10048.

59. Muschitz C, Kocijan R, Haschka J, Zendeli A, Pirker $\mathrm{T}$, Geiger C. et al. The impact of vitamin D, calcium, protein supplementation, and physical exercise on 
bone metabolism after bariatric surgery: the BABS study. J Bone Miner Res 2016; 31(3):672-82. doi: 10.1002/jbmr.2707.

60. Marengo AP, Guerrero Pérez F, San Martín L, Monseny R, Casajoana A, Valera R. et al. Is trabecular bone score valuable in bone microstructure assessment after gastric bypass in women with morbid obesity? Nutrients 2017; 9(12):1314. doi: 10.3390/nu9121314.

61. Hadji P, Aapro MS, Body J-J, Gnant M, Brandi ML, Reginster JY. et al. Management of Aromatase Inhibitor-Associated Bone Loss (AIBL) in postmenopausal women with hormone sensitive breast cancer: Joint position statement of the IOF, CABS, ECTS, IEG, ESCEO, IMS, and SIOG. J Bone Oncol 2017; 7:1-12. doi: 10.1016/j.jbo.2017.03.001.
62. Mariotti V, Page DB, Davydov O, Hans D, Hudis CA, Patil S. et al. Assessing fracture risk in early stage breast cancer patients treated with aromataseinhibitors: An enhanced screening approach incorporating trabecular bone score. J Bone oncol 2016; 7:32-37. doi: 10.1016/j.jbo.2016.10.004.

63. Hong A, Kim J, Lee K, Kim T, Im S, Moon H. et al. Long-term effect of aromatase inhibitors on bone microarchitecture and macroarchitecture in nonosteoporotic postmenopausal women with breast cancer. Osteoporos Int 2017; 28(4):1413-22. doi: 10.1007/s00198-016-3899-6.

64. Catalano A, Gaudio A, Agostino R, Morabito N, Bellone F, Lasco A. Trabecular bone score and quantitative ultrasound measurements in the assessment of bone health in breast cancer survivors assuming aromatase inhibitors. $\boldsymbol{J}$ Endocrinol Invest 2019; 42(11):1337-43. doi: 10.1007/s40618-01901063-0 